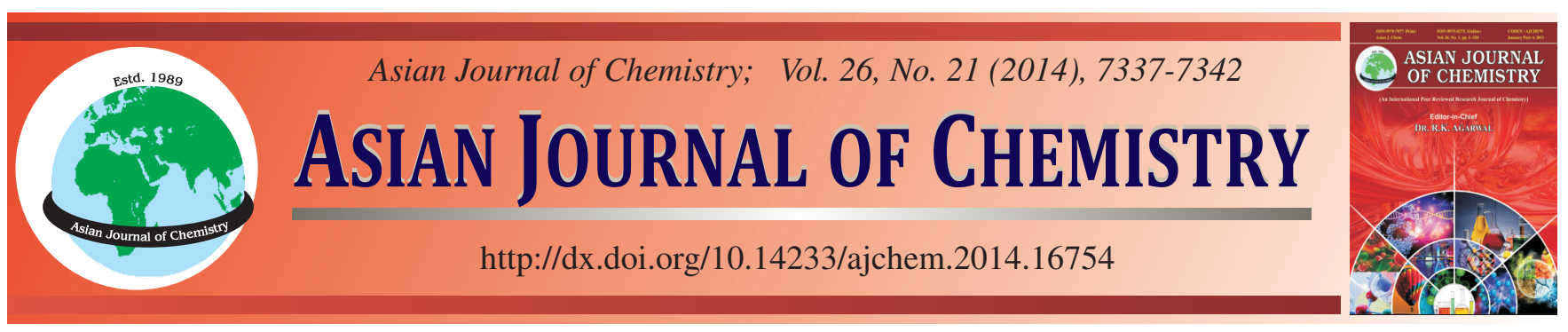

\title{
Synthesis and Swelling Kinetics of Superabsorbent Rice Straw Cellulose Graft Copolymers
}

\author{
Helmiyati ${ }^{*}$, Aser Saefumillah and Wina Yulianti
}

Department of Chemistry, FMIPA, University Indonesia, Depok, Indonesia

*Corresponding author: Fax: +62 21 7863432; Tel: +62 21 7270027; E-mail: helmi-yt@ui.ac.id

\section{INTRODUCTION}

Lately introduced superabsorbent of graft copolymerization of vinyl monomers to natural polymers known as superabsorbent composites, such as chitosan ${ }^{1}, \operatorname{starch}^{2}$, cellu$\operatorname{lose}^{3}$, montmorillonite ${ }^{4}$. Copolymerization conducted to get the desired properties, increase the absorption capacity. Natural polymers used to replace synthetic polymers is more difficult to decompose in the environment and the prices tend to be expensive . $^{5}$.

The agricultural waste such as wheat straw, bagasse, rice straw, cassava dregs and others can be used as a more attractive alternative to get cellulose. Waste of rice straw utilization is not optimal, the farmers remove the rice straw by burning them, as the consequence of increase air pollution. Utilization of rice straw as a superabsorbent is expected to increase the economic value and overcome the environmental pollution. The rice straw including stems, leaves and stalks, at the time the crop was harvested, the straw is parts of plant discarded about $45 \%$ of rice straw produced as the rest of the plant. Traditionally the use of straw is very limited for household and farming activities, such as, animal cage floor mats, animal feed, fuel in the industrial the manufacture of tiles, bricks and organic fertilizer. The use of superabsorbent in agriculture aims to reduce irrigation water consumption and plant mortality rates, increase the availability of water in the soil that allows plants to last longer, reducing the tendency of soil compaction, prevent erosion, increase the fertilizer efficiency and bind heavy metals ${ }^{6}$.

Superabsorbent polymer or hydrogel is a three-dimensional network formed from hydrophilic polymer cross link. Hydrophilic properties of hydrogels are the factors that determine the ability to absorb and retain large amounts of water ten to a thousand fold ${ }^{7}$. The ability of the hydrogel to absorb water increased with increasing hydrophilic groups attached to the polymer main frame. Resilienc of dissolution hydrogel increased with the increasing cross link in the polymer network. Hydrogels are sensitive to environmental changes such as changes in $\mathrm{pH}$, temperature, or the concentration of the environment. Based on the properties of hydrogels are widely used to control the release of bioactive agents in pharmaceutical field and control the absorption and release of water and fertilizer in agriculture ${ }^{6}$.

Huang et al. ${ }^{8}$ have reported the modified bagasse cellulose by grafting copolymerization of acrylic acid, acrylamide monomer with the use of ammonium sulfate redox pair or sodium sulfite as initiator ${ }^{7}$. The resulting superabsorbent polymer used as a slow release control of ammonium and phosphate. Zhang et al. ${ }^{9}$ acrylic acid and acrylamide as monomer grafting to waste of jute yarn that has been chemically modified. Polymerization using ammonium persulfate initiator and the crosslinking agent $N-N$-methylene-bis-acrylamide. Formed polymer is applied as control the absorption and release of urea. 
In this research, the synthesis of cellulose superabsorbent by grafting the rice straw cellulose with acrylic acid, acrylamide monomer on rice straw using initiator of potassium persulphate (KPS) and crosslinking of $N, N$ '-methylene-bisacrylamide. Characteristics of polymer functional groups of superabsorbent rice straw poly(acrylic acid-co-acrylamide) with fourier transform spectroscopy infrared (FTIR), surface morphology with scanning electron microscopy (SEM) and heat stability with differential scanning calorimetry (DSC). Swelling capacity superabsorbent of rice straw cellulose expected can be applied to control slow release of water and fertilizer, by studying the kinetics of swelling.

\section{EXPERIMENTAL}

Rice straw used in this study was obtained from Bogor (Indonesia). Toluene (Merck) used to extract the cellulose in rice straw, hydrogen peroxide (Merck), potassium hydroxide (Merck) was used as an impurity removal rice straw cellulose, acrylic acid, acrylamide (Nippon Shokubai) used as a monomer, potassium persulphate (KPS; Merck) as initiator, $N, N$ '-methylenebis-acrylamide (MBA; Sigma) as crosslinking, ammonium chloride (Merck), potassium dihydrogen phosphate (Merck), urea (Merck) was used as the absorbate.

Cellulose isolation of rice straw: The isolation of rice straw cellulose was performed by the reported method ${ }^{10}$. Rice straw is washed with warm water to remove impurities and water-soluble substances. The rice straw grinded and sieved with 40 mesh sieve. Rice straw powder was extracted with a mixture of toluene:water at ratio of $2: 1$ for $6 \mathrm{~h}$, then straw dried at $50{ }^{\circ} \mathrm{C}$ for $24 \mathrm{~h}$. Hemicellulose is removed by adding $5 \% \mathrm{KOH}$ at room temperature for $24 \mathrm{~h}$ and at $90{ }^{\circ} \mathrm{C}$ for $2 \mathrm{~h}$. Lignin is removed by adding $2 \% \mathrm{H}_{2} \mathrm{O}_{2}$ solution $\mathrm{pH}$ set up to 12 with $4 \% \mathrm{KOH}$ at $40{ }^{\circ} \mathrm{C}$ for $8 \mathrm{~h}$ and $90{ }^{\circ} \mathrm{C}$ for $4 \mathrm{~h}$. The precipitate then washed until neutral and dried at $50^{\circ} \mathrm{C}$ for 24 $\mathrm{h}$. The process of dissolution of rice straw cellulose was as reported $^{11}$. Samples of rice straw cellulose as much as $0.5 \mathrm{~g}$ dissolved in $15 \mathrm{~mL}$ of $7 \%$ sodium hydroxide solution and $12 \%$ urea.

Synthesis of cellulose copolymer with acrylic acid and acrylamide: Synthesis of polymer based on experimental ${ }^{11}$, solution of cellulose fed into the reactor in the form of a three neck flask, reflux condenser and nitrogen hoses. The reactor was placed in a water bath at $65^{\circ} \mathrm{C}$. Oxygen gas is removed from the reactor by nitrogen gas into the reactor for $0.5 \mathrm{~h}$. Potassium persulfate in distilled water introduced into the reactor and the mixture of acrylic acid, acrylamide and $N, N^{\prime}$ methylene-bis-acrylamide in distilled water fed into the reactor. Polymerization process kept $2 \mathrm{~h}$ at $65{ }^{\circ} \mathrm{C}$. Obtained superabsorbent were washed in distilled water, ethanol and acetone for $24 \mathrm{~h}$ at room temperature. Superabsorbent was cut into pieces of approximately $0.5 \mathrm{~cm}$. Then dried at $65{ }^{\circ} \mathrm{C}$ until constant weight. Experiments were carried out with a variation on cellulose, potassium persulphate (KPS), acrylic acid (AA), acrylamide (AM) and $N, N^{\prime}$-methylene-bis-acrylamide and neutralization of acrylic acid with sodium hydroxide to the sample code Gel superabsorbent (GS) from GS 01 to GS 16.

Characterization: Superabsorbent function groups were analyzed by FTIR at wave numbers 4000 to $400 \mathrm{~cm}^{-1}$ using
KBr pellets. Analysis of the surface morphology by SEM type JSM 5000 with a magnification of 500 and 10000 times. The thermal resistance with differential scanning colorimetry (DSC).

Efficiency of grafting: The efficiency of grafting is the ratio the mass grafted of monomers on cellulose with the initial monomers. Efficiency of grafting can be calculated by the following eqn.

Efficiency of grafting $=\frac{\text { superabsorbent mass }- \text { sellulose mass }}{\text { initial monomer mass }} \times 100 \%$ (1)

Capacity of swelling: Measurement of swelling capacity of the absorbate, based on previous work ${ }^{11}$, by weighing the dry gel $(0.1 \mathrm{~g})$ in a nylon cloth immersed into distilled water, at room temperature to reach equilibrium. Superabsorbent separated from unabsorbed water. Swelling capacity $\left(S_{e}\right)$ is calculated with the following eqn.

$$
\mathrm{S}_{\mathrm{e}}=\frac{\mathrm{m}_{2}-\mathrm{m}_{1}}{\mathrm{~m}_{1}}
$$

$\mathrm{m}_{1}$ is the mass of dry gel, $\mathrm{m}_{2}$ is the mass absorption of the gel after swelling. Experiment carried out on distilled water, urea, ammonium chloride and potassium dihydrogen phosphate.

\section{RESULTS AND DISCUSSION}

Synthesis of superabsorbent copolymers rice straw cellulose graft poly (acrylic acid co acrylamide): The success of the synthesis of graft copolymers can be seen from the grafting efficiency shown in Fig. 1.

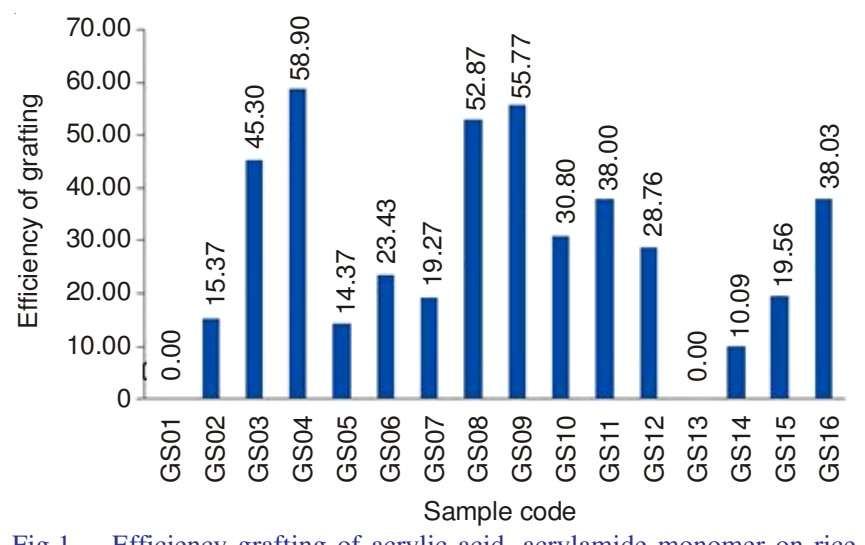

Fig.1. Efficiency grafting of acrylic acid, acrylamide monomer on rice straw cellulose

Based on Fig. 1, the high efficiency can be seen in the order GS04, GS09 dan GS08 arranged by concentration that is $13915 \mathrm{mmol} / \mathrm{L}$. The increase of $N, N^{\prime}$-methylene-bisacrylamide amount added increase the efficiency of grafting. Small amount of $N, N^{\prime}$-methylene-bis-acrylamide caused no formation crosslink so that the copolymer not occur, can be seen at GS01 and GS13 superabsorbent not formed, caused GS01 and GS13 formula are made with the smallest $N, N^{\prime}$ methylene-bis-acrylamide of concentration is $2.319 \mathrm{mmol} / \mathrm{L}$. The $N, N$ '-methylene-bis-acrylamide is a crosslinking agent that links the polymer chains to each other to form cross link.

Mechanism of copolymerization with potassium persulphate initiator, acrylic acid, acrylamide monomer and $N, N^{\prime}$ methylene-bis-acrylamide crosslinking, involved several stage 
e.g., initiation, propagation and termination. The initial stage of the reaction is the formation of the radical anion of potassium persulphate to form sulphate radicals caused by heating. Sulfate anion radical formed at $60-75{ }^{\circ} \mathrm{C}^{5,12}$.

The initiation stage of sulfate anion radicals attack the hydrogen of the hydroxyl group bound to the cellulose and take the hydrogen atom to form the radical cellulose. Propagation stage begins when the radical formed in the initiation step reacts with other monomers to form macromolecules radical. Stage of propagation continuously until the monomer is consumed. The final stage is termination can take place in two ways that is the combination and disproportionation ${ }^{12}$.

Stage combination take place when the radical reacts with acrylic acid-acrylamide cellulose radicals with others in order to obtain long chains, whereas the disproportionation stage, each acrylic acid-acrylamide cellulose radicals when it reaches a critical chain length termination will take place. Disproportionation stage involves the transfer of an atom, usually hydrogen chain from one end to the other end of the chain. Possible termination stage that occurs depends on the structure of chain end acrylic acid-acrylamide cellulose radicals that formed. Factors affecting the termination stage are steric repulsion, polar groups repulsion such as ester groups can increase the activation energy resulting the termination of the combination.

By using the crosslinking $N, N^{\prime}$-methylene-bis-acrylamide can lower the activation energy so that the macromolecular radicals react to form graft copolymer superabsorbent straw with the long chain. Sulfate anion radicals not reacted with the cellulose can initiating acrylamide and acrylic acid to form acrylamide and acrylic acid copolymers form a homopolymer of acrylamide and acrylic acid homopolymer. Hydrogel washing process aims to eliminate water-soluble homopolymer, unreacted monomers, crosslinking agent, initiator and other impurities ${ }^{13}$.

\section{Characterization of Superabsorbent}

Absorption spectrum with FTIR: FTIR spectrum superabsorbent is presented in Fig. 2. Absorption spectrum of rice straw cellulose ( $2 a)$, absorption spectrum of the copolymerization of acrylic acid with acrylamide (2b) and absorption spectrum superabsorbent of rice straw poly (acrylic acid co acrylamide) graft copolymer (2c). Wide absorption in $3500-3100 \mathrm{~cm}^{-1}$ show superabsorbent rice straw cellulose poly (acrylic acid-co-acrylamide) and copolymer of acrylic acidacrylamide showed $\mathrm{NH}$ stretching vibration of acrylamide that overlap with acrylic acid $\mathrm{OH}$ stretching vibration. Absorption at $2900 \mathrm{~cm}^{-1}$ is the $\mathrm{C}-\mathrm{H}$ stretching vibration. Absorption at $1728 \mathrm{~cm}^{-1}$ shows the $\mathrm{C}=\mathrm{O}$ stretching in acrylic acid. Absorption at $1666 \mathrm{~cm}^{-1}$ shows the $\mathrm{C}=\mathrm{O}$ stretch shifts due to the superposition of the amide group on the wave number 1659 $\mathrm{cm}^{-1}$ and group $\mathrm{C}=\mathrm{O}$ in $\mathrm{COOH}$ at wave number $1718 \mathrm{~cm}^{-1}$. In the wave number $1575 \mathrm{~cm}^{-1}$ is an amide absorption characteristics. C-O-C stretching vibration spectra of superabsorbent cellulose (2c) at a wavelength of 1173 to $1057 \mathrm{~cm}^{-1}$ shows the absorption spectrum is wider than the copolymer (2b).

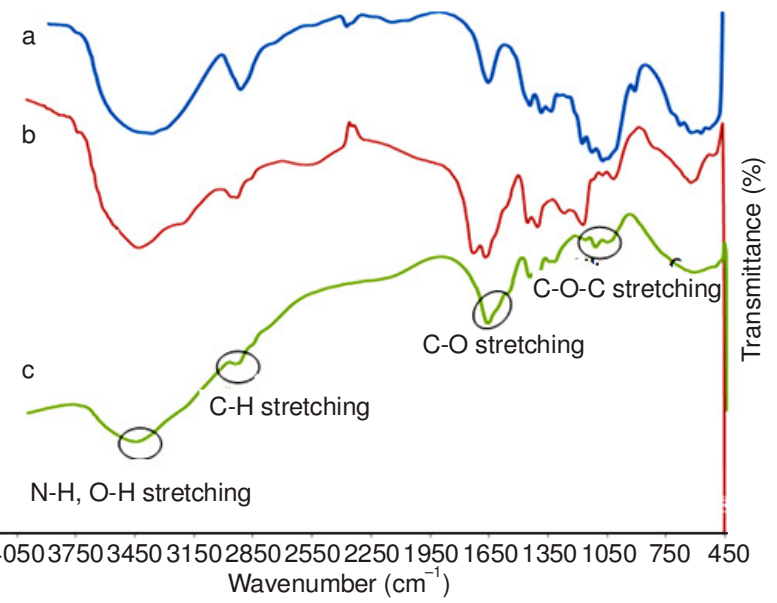

Fig. 2. FTIR spectrum of the cellulose (a) copolymer acrylate acidacrylamide (b) and superabsorbent of cellulose (c)

Analysis of the surface morphology by SEM: The process of grafting has been formed is comparing the micrographs superabsorbent of the rice straw cellulose poly (acrylic acidco-acrylamide) graft copolymer with micrographs of the cellulose and copolymers acrylic acid-acrylamide, can be seen in Fig. 3.

Micrograph in Fig. 3a is copolymer acrylic acidacrylamide magnification 500 times, copolymers tend to be straight with relatively little branching when compared with superabsorbent of rice straw cellulose poly (acrylic acid-coacrylamide) in Fig. 3c. This indicates the grafting of acrylic acid and acrylamide has occurred on the rice straw cellulose. The pores are formed on the copolymer of acrylic acidacrylamide are visible by magnification 7500 times with smooth texture, in Fig. 3b. The texture of superabsorbent of rice straw cellulose more rude, this is due to the grafting so that the monomer to the cellulose, superabsorbent network formed more tightly in Fig. 3d.
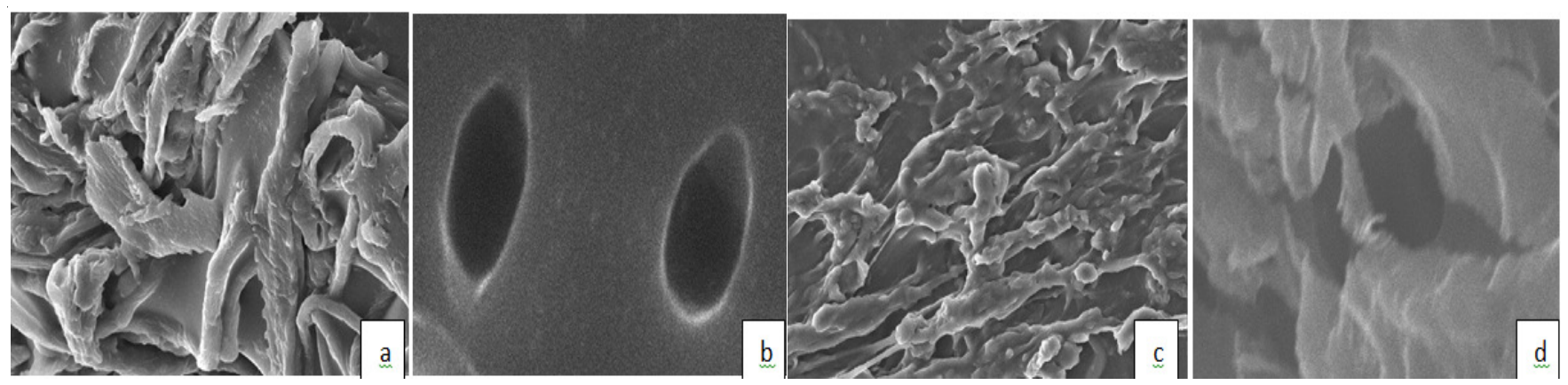

Fig. 3. Micrographs of copolymers acrylic acid-acrylamide magnification 500 times (a) 7500 times (b) superabsorbent of cellulose 500 times (c) and 5000 times $(\mathrm{d})$ 
Thermal analysis of superabsorbent: Thermal resistance of rice straw cellulose is presented in Fig. 4a. Rice straw cellulose has one endothermic peak and one exothermic peak. Endothermic process occurs at temperatures between 33.10 to $105{ }^{\circ} \mathrm{C}$ with a peak at $93.80{ }^{\circ} \mathrm{C}$. This indicates rice straw cellulose materials have lost physically water bound at that temperature. The water loss is common in materials containing cellulose $^{14}$. The amount of the rate of energy absorbed to the endothermic process is $-5.15 \mathrm{~mW}$. Rice straw cellulose pyrolysis occurs at temperatures between 361.66 to $388.68{ }^{\circ} \mathrm{C}$ with a peak of $368.22{ }^{\circ} \mathrm{C}$, it shows the cellulose decomposition process ${ }^{15}$. The amount of energy released rate for the pyrolysis process is $2.32 \mathrm{~mW}$ (Fig. 4a)
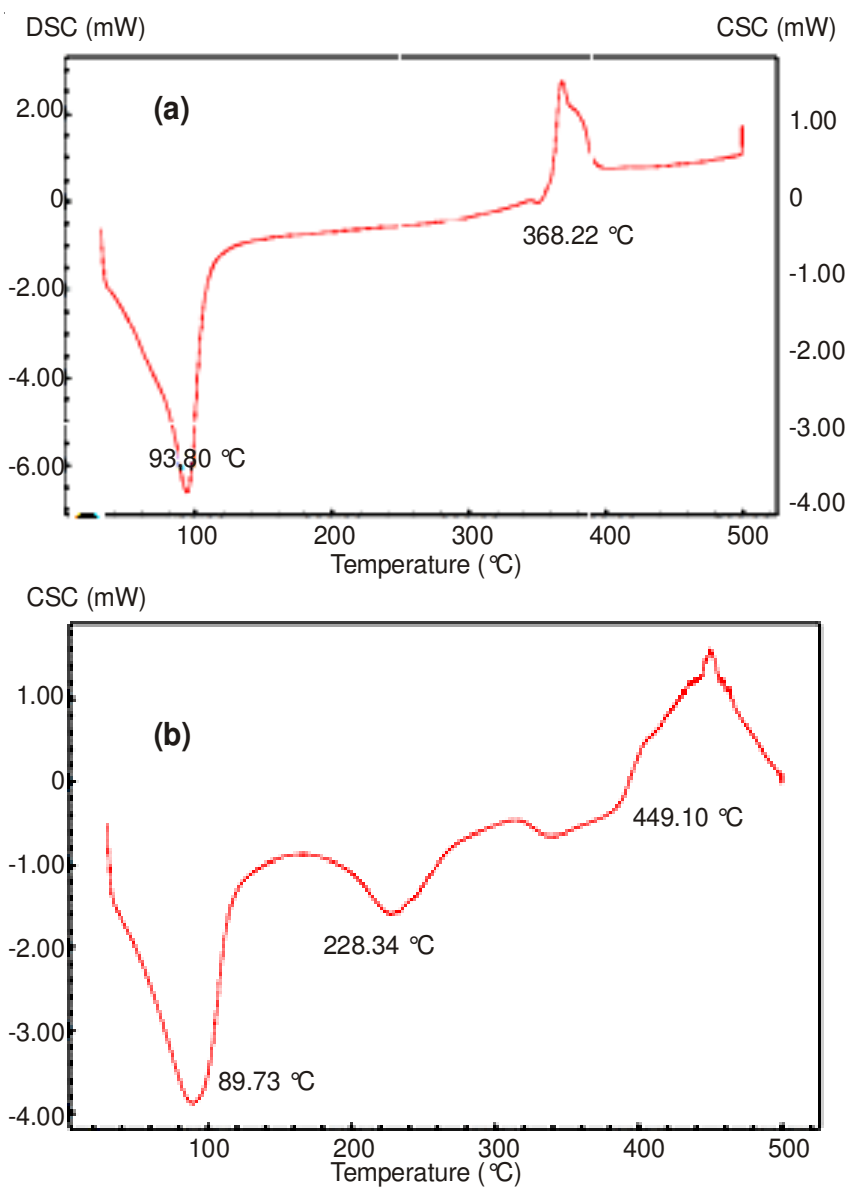

Fig. 4. DSC thermogram of rice straw cellulose (a), superabsorbent of rice straw cellulose

DSC results for superabsorbent of rice straw poly (acrylic acid-co-acrylamide) graft copolymer showed two endothermic process between 97.27 to $112.73{ }^{\circ} \mathrm{C}$ with peak at $89.73{ }^{\circ} \mathrm{C}$ and between 204.26 to $254.72{ }^{\circ} \mathrm{C}$ with peak at $228.34{ }^{\circ} \mathrm{C}$. The amount of the rate of energy absorbed for both the endothermic process is -0.42 and $-1.07 \mathrm{~mW}$. This indicates that superabsorbent material have lost physically bound water on both the temperature. The loss mass of physically bound water in this material is smaller than rice straw cellulose material. Pyrolysis at this superabsorbent occurs at temperatures between 447.49 to $465.10^{\circ} \mathrm{C}$ with peak at $449.10^{\circ} \mathrm{C}$. The amount of energy released rate for the pyrolysis process is $1.53 \mathrm{~mW}$ (Fig. 4b).
Swelling capacity: Determination of swelling capacity done by measuring weight of the superabsorbent after the swelling process, the absorbate are distilled water, urea, ammonium chloride and potassium dihydrogen phosphate. The results of the swelling capacity of superabsorbent all samples can be seen in Fig. 5.

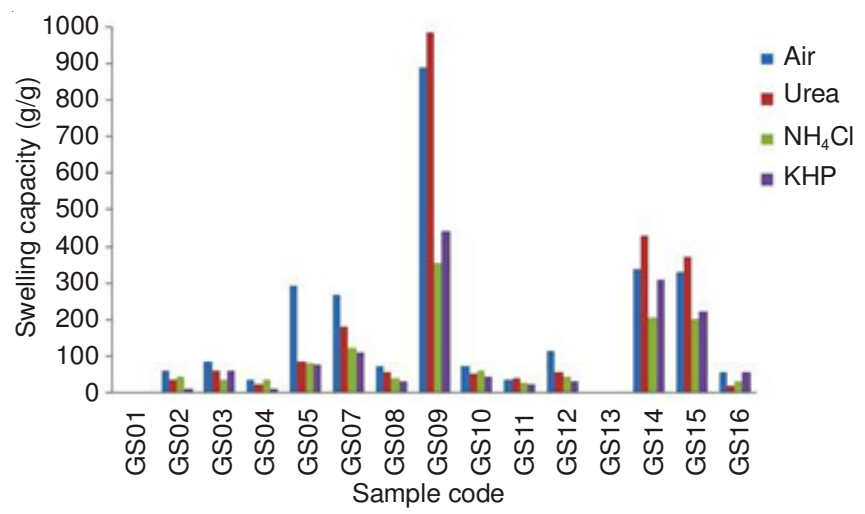

Fig. 5. Swelling capacity of superabsorbent rice straw cellulose poly (acrylic acid-co-acrylamide) graft copolymer

Based on Fig. 5 superabsorbent GS09 have highest swelling capacity of the water, urea, ammonium chloride and potassium dihydrogen phosphate. Swelling capacity values of water is $895.48 \mathrm{~g} / \mathrm{g}$. Value of swelling capacity of urea is 986.72 $\mathrm{g} / \mathrm{g}$; ammonium chloride is $387.11 \mathrm{~g} / \mathrm{g}$ and potassium dihydrogen phosphate is $448.98 \mathrm{~g} / \mathrm{g}$. Swelling capacity of the urea solution showed the highest value compared to water. Urea is a neutral molecule that have hydrophilic group $\mathrm{NH}_{2}$. The addition of urea in solution causing the increasing hydrogen bonding interactions between the superabsorbent and solutions that causing greater swelling capacity ${ }^{5,16}$. According to Sadeghi et al. ${ }^{17}$ hydrogel swelling capability will be reduced with the increasing number of ions that decompose in water.

Parameter of rate based Voigt equation model: Preliminary study of the swelling kinetics of rice straw cellulose the graft poly (acrylic acid co-acrylamide) using Voigt-based model by the following eqn.

$$
\mathrm{S}_{\mathrm{t}}=\mathrm{S}_{\mathrm{e}}\left(1-\mathrm{e}^{-\mathrm{t} / \tau}\right)
$$

$\mathrm{S}_{\mathrm{t}}$ is Swelling at time $\mathrm{t}, \mathrm{S}_{\mathrm{e}}$ is swelling at equilibrium and $\mathrm{t}$ is the rate parameter. Parameters of rate based on the Voigt equation model can be used as measure swelling rate, which is parameter low rate indicating speed of high swelling. Conversely, if large rate parameter indicates the speed of the slow swelling. Rate parameters can be determined by plot of the $\ln \left(1-\mathrm{S}_{\mathrm{t}} / \mathrm{S}_{\mathrm{e}}\right)$ as function of time. The graph show $\ln \left(1-\mathrm{S}_{\mathrm{t}} / \mathrm{S}_{\mathrm{e}}\right)$ versus time for the absorbate of water, solution of urea, ammonium chloride and potassium dihydrogen phosphate presented in Fig. 6.

Based on Fig. 6, it can be seen of urea solution have slope value $\left(R^{2}\right)$ the largest, the value of the slope is inversely proportional to the rate parameters in the equation Voight, if the slope is large then the rate parameter indicates the value of small so that speed of high swelling. Parameters swelling rate of superabsorbent in water is 746 , urea solution is 560 , potassium dihydrogen phosphate is 1677 and ammonium chloride is 


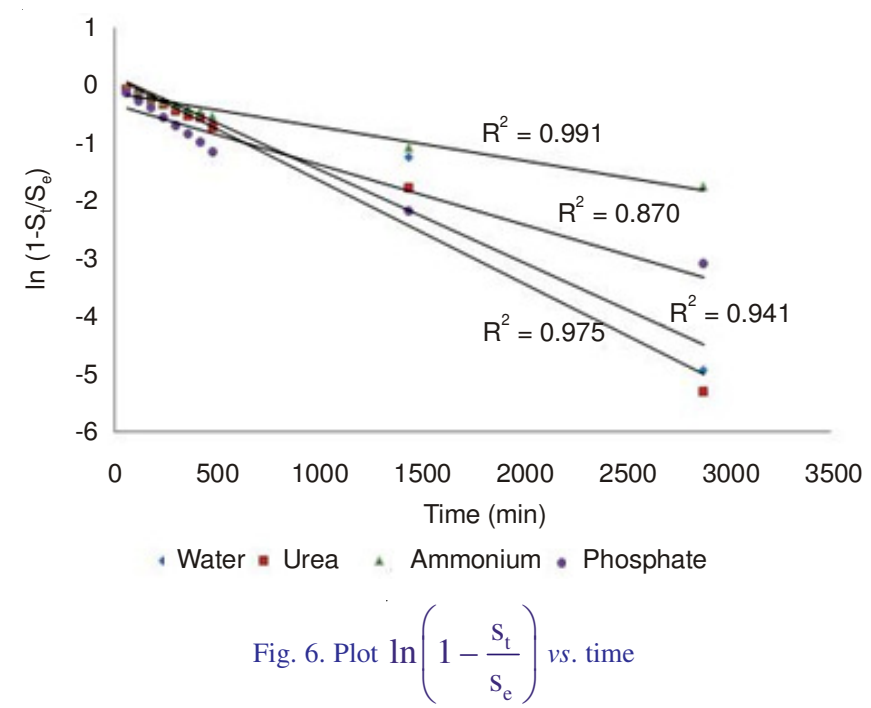

1768. Based on the data of rate parameters are obtained, the order the speed of the fastest superabsorbent swelling is urea, water, potassium dihydrogen phosphate and ammonium chloride, this results support the data of swelling capacity.

Swelling kinetics of superabsorbent: Capacity of swelling as a function of time from rice straw cellulose graft superabsorbent poly (acrylic acid co-acrylamide) to water, urea, ammonium and potassium dihydrogen phosphate presented in Fig. 7, it can be seen that the swelling capacity increases exponentially with time. At the initial time of the swelling capacity superabsorbent increase and then swelling capacity with time tends slowed to near equilibrium.

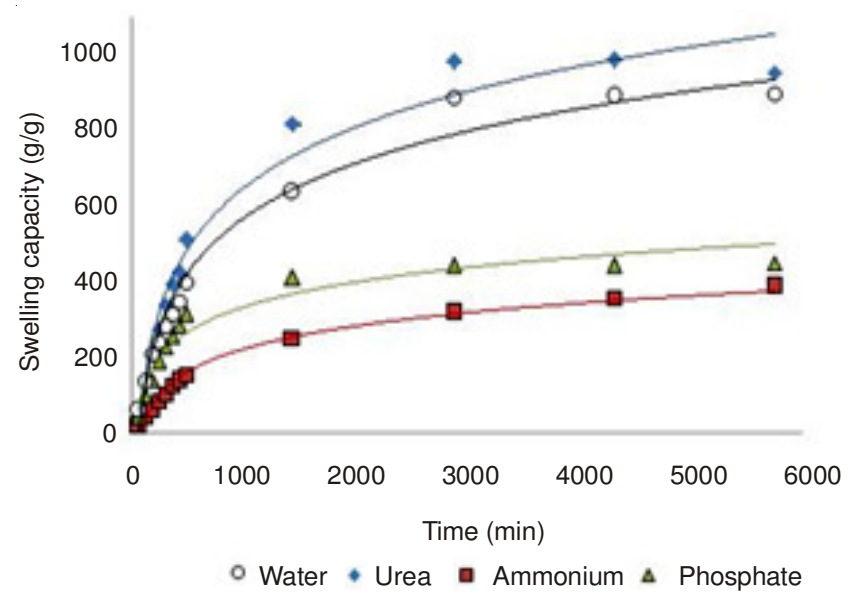

Fig. 7. Plot swelling capacity versus time

The addition of ammonium and phosphate salts to water will decrease the osmotic pressure difference. This can be seen in Fig. 7. The ability swelling of superabsorbent in ammonium chloride and potassium dihydrogen phosphate solution is lower than the swelling ability in water and urea solution. The swelling process due to the osmotic pressure difference between the superabsorbents with solution. The higher the osmotic pressure difference causes high swelling capacity ${ }^{18}$.

Determination of swelling reaction order: Determination of the reaction order tested using first reaction order and second reaction order kinetics equation, kinetics equation of first reaction order shown in eqn.

$$
\ln \frac{\mathrm{S}_{\mathrm{e}}}{\left[\mathrm{S}_{\mathrm{e}}-\mathrm{S}_{\mathrm{t}}\right]}=\mathrm{kt}
$$

Kinetics equation of second reaction order shown in eqn.

$$
\frac{1}{\mathrm{~S}_{\mathrm{t}}}=\frac{1}{\mathrm{~S}_{\mathrm{e}}}+\frac{1}{\mathrm{~S}_{\mathrm{e}}^{2} \mathrm{kt}}
$$

The first reaction order can be obtained by plot $\ln \frac{\mathrm{S}_{\mathrm{e}}}{\left[\mathrm{S}_{\mathrm{e}}-\mathrm{S}_{\mathrm{t}}\right]}$ to time (Fig. 8a) and second reaction order is obtained by plot the $1 / \mathrm{S}_{\mathrm{t}}$ to $1 / \mathrm{t}$ (Fig. $8 \mathrm{~b}$ ).
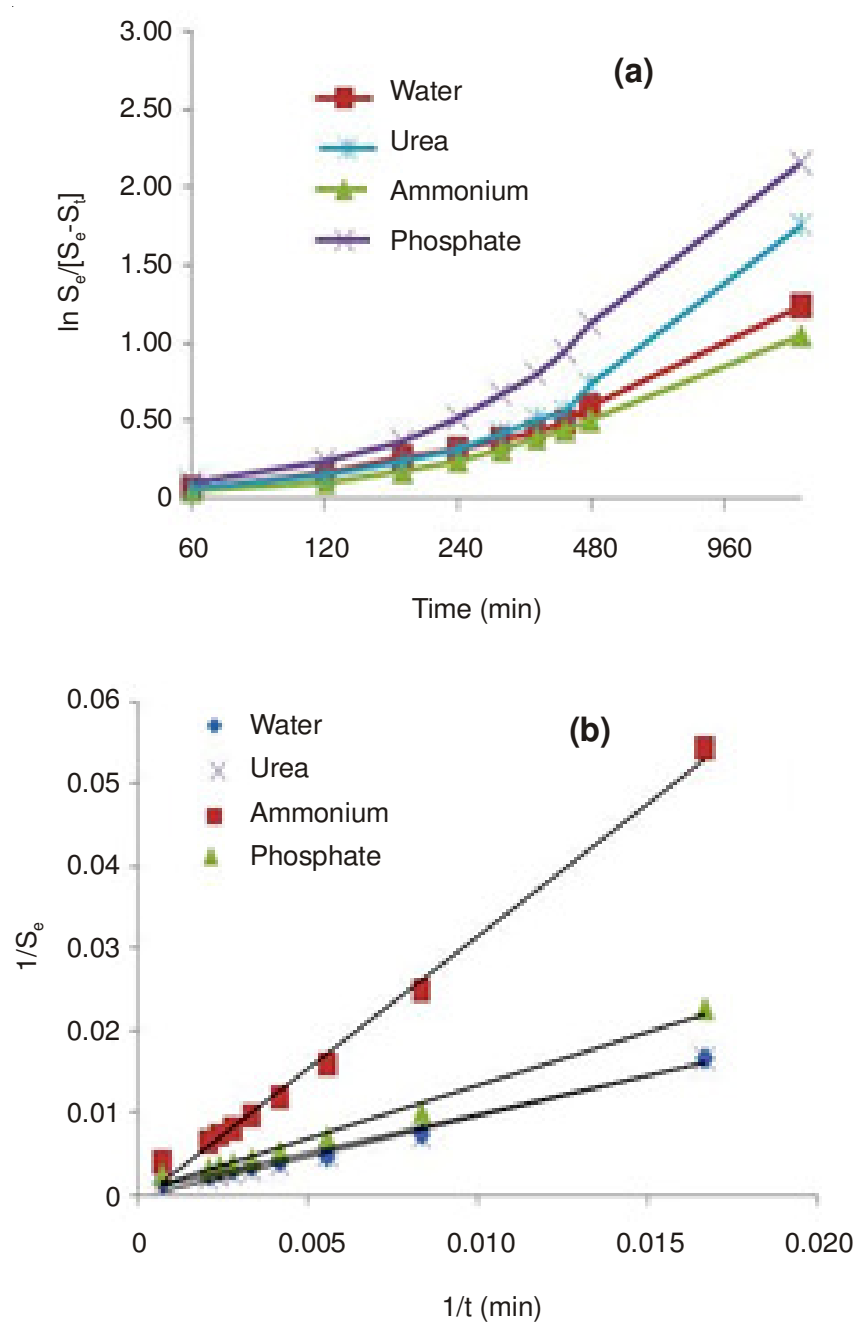

Fig. 8. Plot $\ln \frac{\mathrm{S}_{\mathrm{e}}}{\left[\mathrm{S}_{\mathrm{e}}-\mathrm{S}_{\mathrm{t}}\right]}$ to time (a) and $1 / \mathrm{S}_{\mathrm{t}}$ to $1 / \mathrm{t}$ (b)

The value of the regression coefficient $\left(\mathrm{R}^{2}\right)$ of the curve can be seen in Table-1

\begin{tabular}{ccc}
\multicolumn{3}{c}{ TABLE-1 } \\
& \multicolumn{2}{c}{ REGRESSION COEFFICIENTS OF THE } \\
& FIRST AND SECOND REACTION ORDER \\
\hline Absorbat & First order $\left(\mathrm{R}^{2}\right)$ & Second order $\left(\mathrm{R}^{2}\right)$ \\
\hline Water & 0.935 & 0,988 \\
Urea & 0.887 & 0.990 \\
Phosphate & 0.878 & 0.989 \\
Ammonium & 0.933 & 0.994 \\
\hline
\end{tabular}


Based on Fig. 8 and Table-1, the value of the regression coefficient $\left(\mathrm{R}^{2}\right)$ obtained from second-order kinetics for all absorbate is greater than a first-order reaction kinetics and it can be concluded that the rate of swelling absorbate from water, solution of urea, ammonium chloride and potassium dihydrogen phosphate on superabsorbent fit to second order rate law.

The law of reaction rate of absorbate swelling to water, solution of urea, ammonium chloride and potassium dihydrogen phosphate on superabsorbent can be written with equation , It was indicated the rate of swelling superabsorbent rice straw cellulose graft poly (acrylic acid co-acrylamide) is proportional to the square concentration of the absorbate.

\section{Conclusion}

Superabsorbent of cellulose graft poly (acrylic acid-coacrylamide) from rice straw cellulose has been synthesized successfully and characterized by FTIR, SEM and DSC. Swelling capacity of superabsorbent having the highest value is GSO9 to water is $895.78 \mathrm{~g} / \mathrm{g}$ and urea, potassium dihydrogen phosphate and ammonium chloride are 986.72, 448.98 and $387,11 \mathrm{~g} / \mathrm{g}$, respectively. Parameters swelling rate of superabsorbent in water is 746 , solution of urea 560 , potassium dihydrogen phosphate is 1677 and ammonium chloride is 1768, based on data swelling capacity and rate parameters are obtained, the order of the speed of the fastest swelling superabsorbent are urea, air, potassium dihydrogen phosphate and ammonium chloride. Kinetics of swelling superabsorbent with adsorbate water, urea, potassium dihydrogen phosphate and ammonium chloride fit the second-order kinetics.

\section{ACKNOWLEDGEMENTS}

This research was supported by Research Fund Directorate of Research and Community Service, University of Indonesia, for fiscal year 2013.

\section{REFERENCES}

1. C. Spagnol, F.H.A. Rodrigues, A.G.B. Pereira, A.R. Fajardo, A.F. Rubira and E.C. Muniz, Carbohydr. Polym., 87, 2038 (2011).

2. K. Zhong, Z.T. Lin, X.L. Zheng, G.B. Jiang, Y.S. Fang, X.Y. Mao and Z.W. Liao, Carbohydr. Polym., 92, 1367 (2013).

3. S. Pan and A.J. Ragauskas, Carbohydr. Polym., 87, 1410 (2012).

4. Y. Bao, J. Ma and Y. Sun, Carbohydr. Polym., 88, 589 (2012).

5. R. Liang, H. Yuan, G. Xi and Q. Zhou, Carbohydr. Polym., 77, 181 (2009).

6. J. Mohammad, Z. Mehr and K. Kabiri, Iran. Polym. J., 17, 451 (2008).

7. Z. Ma, Q. Li, Q. Yue, B. Gao, W. Li, X. Xu and Q. Zhong, Chem. Eng. J., 171, 1209 (2011).

8. Z.Q. Huang, X.L. Xie, Y. Chen, J.P. Lu, Z.F. Tong and C.R. Chimie, C. R. Chim., 11, 73 (2008).

9. Y. Zhang, F. Wu, L. Liu and J. Yao, Carbohydr. Polym., 91, 277 (2013).

10. P. Lu and Y.-L. Hsieh, Carbohydr. Polym., 87, 564 (2012).

11. F. Wu, Y. Zhang, L. Liu and J. Yao, Carbohydr. Polym., 87, 2519 (2012).

12. J. Physiol. Sci., 21, 39 (2010).

13. W.A. Laftah, S. Hashim and A.N. Ibrahim, Polymer-Plastics Technol. Eng., 50, 1475 (2011).

14. S. Zhang, F.-X. Li, J.- Yu and Y.-L. Hsieh, Carbohydr. Polym., 81, 668 (2010).

15. M.L. Rosa, N. Rehman, M.I.G. de Miranda, S.M.B. Nachtigall and C.I.D. Bica, Carbohydr. Polym., 87, 1131 (2012).

16. Y. Zhang, F. Wu, L. Liu and J. Yao, Carbohydr. Polym., 91, 277 (2013).

17. M. Sadeghi, J. Sci. I.A. Univ., 17, 19 (2007)..

18. O. Okay, Hydrogel Sens. Actuators, Springer Series on Chemical Sensors and Biosensors, 6, 1 (2009). 